\title{
Lipid patterns of edible fungi
}

\section{TÖRLEY and E. VADON-GYÖREY}

\begin{abstract}
TÖRLEY, D. \& VADON-GYÖREY, E. 1978; Lipid patterns of edible fungi. - Karstenia 18 (suppl.). In the course of the investigation of the lipids of fungi by column chromatography several neutral and poral lipid classes have been separated which were analyzed by TLC. The distribution of the lipid classes and the number of components in the fractions showed characteristic differences in the investigated mushroom species. Even members of the same fungus family differ from each other in the lipid composition. Several indicators were used to visualize the spots: iodine vapour, Dragendorff reagent, Liebermann-Burchard reused to visualize the spots: iodine vapour, Dragendorff reagent, Liebermann-Burchard re-
agent, ninhydrine solution, Hanes reagent, iron-sulfosalicylic acid reagent, uV radiation.

D. Törley \& E. Vadon-Györey, Department of Biochemistry and Food Technology, Technical University Budapest, Müegyetem rkp $3 \mathrm{H}-1111$ Budapest, Hungary.
\end{abstract}

\section{Introduction}

The literature dealing with fungal lipids is by means complete. At the beginning of the century Landsiedel \& Bamberger (1), Rosenthal (2), Zellner and his co -workers (3, 4, 5) isolated sterols and cerebrosides from several fungus species. After a long pause the research on the lipids of fungi has continued in the past two decades, so that in recent years considerable information on fungal lipids has accumulated. Brennan \& co-workers (6) reviewed this topic in 1975. Though with the introduction of the thin-layer chromatography and infrared and mass spectrometry extensive lipid analysis of fungi has started, the research is far from being finished. The published results show that in these investigations some lipid classes received more attention than others, and the information is not yet complete. In Hungary the lipids of edible fungi had not been investigated earlier, therefore we began only two years ago this research work on cultivated and wild mushrooms in Hungary. We started with the search for suitable methods for the isolation and identification of lipid classes.

\section{Materials and methods}

The cultivated mushrooms were provided by the trade and the wild growing species were collected in 1976 in the neighbourhood of Budapest.

The mushrooms were homogenized with a double volume of acetone (w/2 v) and filtered with suction. After rehomogenizing with chloroform-methanol 2:1, the combined acetone and the combined chloroform -methanol filtrates were concentrated to dryness. The residue was shaken after the addition of chloroform -methanol-water 80:40:30 according to Folch et al.
(7). Two phases were formed; the upper phase was discarded and the same amount of fresh upper phase was added. After mixing, the lower phase was concentrated to dryness and the residue served as sample for the investigations.

The isolation of the individual lipid classes was performed by different chromatographic techniques. The identification was made partly with standards, partly on the basis of data in the literature.

Analytical thin-layer chromatography (TLC) was performed on $20 \times 20 \mathrm{~cm}$ glass plates using Kieselgel G (according to Stahl). The best solvent systems were chosen by experiments. For the isolation of neutral lipids the n-hexane-diethylether-acetic acid 80:20:1 system was best suited. In the case of polar lipids chloroform-methanol-water $65: 25: 4$ and $65: 25: 2$ systems were used after saturating the vessel at $25^{\circ} \mathrm{C}$ for 1 hour. The detection of the lipid classes was performed with iodine vapour (all lipids) and with the following spray reagents: modified Liebermann-Burchard reagent for sterols, ninhydrine solution for free amino groups, Dragendorff reagent for other nitrogen-containing compounds, reagent of Hanes for phosphate esters, orcinol reagent for carbohydrates, resorcinol reagent for carbohydrates.

\section{Results and discussion}

The separation of the polar and neutral lipids was performed by column chromatography. Kieselgur G colum was prepared with chloroform and the residue of the mushroom extraction, dissolved in chloroform, was applied to it. The column was eluted successively with chloroform (neutral lipids) and methanol (polar lipids). In the similar way a Kieselgur G column was prepared with 
benzene and the elution was performed with benzene and acetone. The eluates were evaporated to dryness at room temperature. The fractions obtained on the chloroform and benzene columns were compared by TLC. No difference was found in the number and the $R_{f}$-values of the components. At the start line the spots appeared very crowded; for better isolation the development was repeated after drying at room temperature.

Better separation of the spots with one development on TLC plates was obtained by the use of silicic acid column (Silicic acid Koch \& Light, 325 mesh). The chloroform solution of the residue of the extraction of Clitocybe nebularis was applied to the column prepared with chloroform. The elution with chloroform yielded two sharply separated neutral lipid fractions ( 1 a and $1 \mathrm{~b}$ ). The polar lipids were eluted with methanol (fraction 2).

Fig. I shows the thin-layer chromatography plate (neutral lipids) treated with iodine vapour indicator. In Fig. 2 the same chromatogram is shown after detection with the Liebermann-Burchard reagent, which was slightly modified by us. The green spots of the sterols appear instantly, in fraction 1 a there may be seen a yellow fluorescent spot, which appears also in the control substance ergosterol.

Fig. 1. Isolation of neutral and polar lipids of critocybe nebuzaris on silicic acid column. I. TLC of neutral lipids. Indicator: iodine vapour. Spotted from left to right: fraction 1 a, ergosterol, cholesterol, stearic acid, fraction 1 b, extraction residue.

Fig. 2. Isolation of neutral and polar lipids of Clitocybe nebularis on silicic acid column. I. TLC of neutral lipids. Indicator: Liebermann reagent.

Spotted from to right: fraction 1 a,ergosterol, cholesterol, stearic acid, fraction $1 \mathrm{~b}$, extraction residue.

0 0

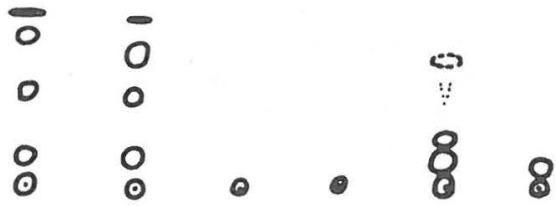

Fig. 3 shows the chromatogram of the extraction residue of $C$. nebularis, soya lecithin, fraction 2 (i.e. polar lipids) of the mushroom eluted from the silicic acid column in 3 different concentrations, and soya phosphatide standard after detection with

Fig. 3. Isolation of neutral and polar lipids of clitocybe nebularis on silicic acid column. I. TLC of neutral lipids. Indicator: iodine vapour.

Spotted from left to right: extraction residue, soya lecithin, fraction 2 (in three concentrations), soya phosphatide.

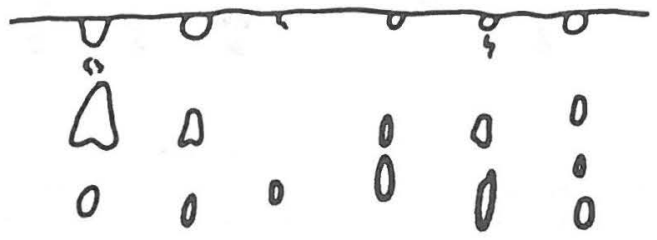

6

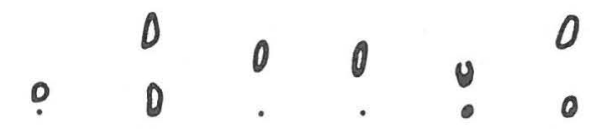


iodine vapour. In Fig. 4 the same chromatogram is shown after detection with orcinol reagent. The well separated three spots are sterol glycosides and cerebrosides. In Fig. 5 the same chramatogram is shown after detection with ammonium molybdate (Hanes reagent). (In a later experiment the spots of the mushroom extract were separated into several components by column chromatography).

To compare more clearly the occurrence of the free amino groups of ethanolamine and the other nitrogen-containing compounds, the thin-layer chromatogram was sprayed successively with two reagents: the left part with ninhydrine, the right part with Dragendorff reagent (Fig. 6). The chromatogram shows that $c$. nebuzaris contains only very small amounts of phosphatidylethanolamine.

Fig. 4. Isolation of neutral and polar lipids of Clitocybe nebularis on silicic acid column. II. TLC of polar lipids.

Spotted from left to right: extraction residue, soya lecithin, fraction 2 (in three concentrations), soya phosphatide.
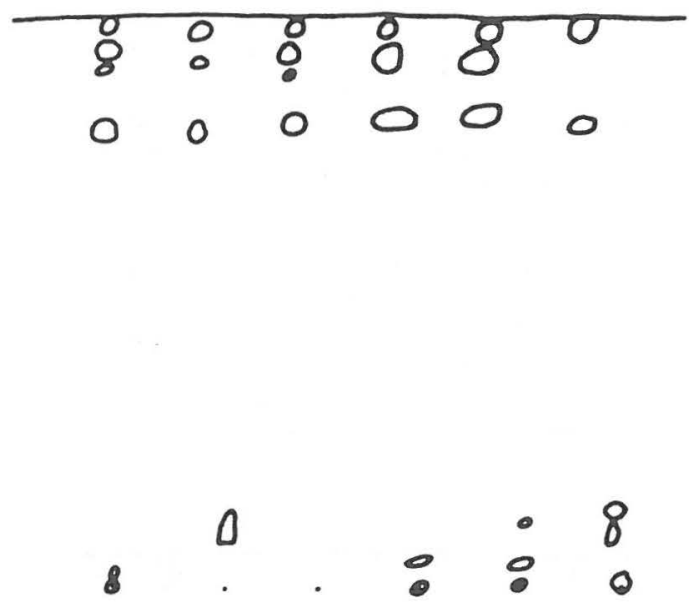

To obtain clearer isolation of the lipid classes, experiments were made with preparative thin-layer chromatography. The extraction residue of the mushrooms was applied to the plate in the form of a band on the start line, and after developing the detection was performed with iodine vapour. The isolated bands were scraped off, and after the sublimation of the iodine the lipid fractions were eluted from the silica gel with chloroform-methanol-water $(1: 2: 0.8)$. The evaporated material was examined by analytical TLC. By this method the lipids of the cultivated mushroom Agaricus bisporus could be separated in the first step into 8 fractions, from which a further $4-5$ spots were isolated by analytical TLC.
Fig. 5. Isolation of neutral and polar lipids of clitocybe nebularis on silicic acid column. II. TLC of polar lipids.

Spotted from left to right: extraction residue, soya lecithin, fraction 2 (in three concentrations), soya phosphatide.
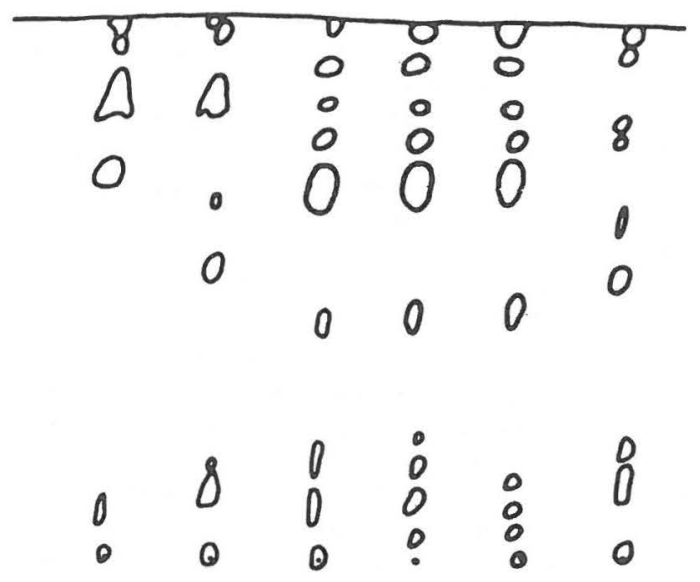

Fig. 6. Isolation of neutral and polar lipids of clitocybe nebularis on silicic acid column. II. TLC of polar lipids. Spotted from left to right: fraction 2, extraction residue, soya lecithin, soya phosphatide.

Indicator: ninhydrine.

Fraction 2, extraction residue, soya lecithin, soya phosphatide.

Indicator: Dragendorff reagent.

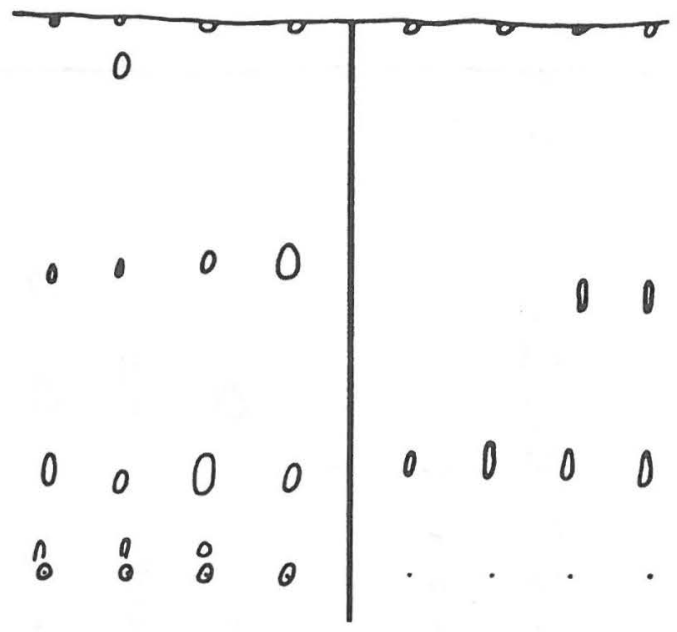


Better separation. was obtained with the use of Florisil columns (60-100 mesh). The sample was applied to a chloroform column and the neutral lipids were eluted with chloroform (fraction 1). By changing successively the polarity of the solvent, sterol glycosides and their esterified products as well as cerebrosides are obtained with chloroform-acetone $1: 1$ (fraction 2). With acetone cerebrosides, mono- and digalactosylglycerides (fraction 3), with chloroform-methanol 9:1 phosphatidic acids are eluted (fraction 4).Chloroform-methanol 1:1 yields phosphatidylserine, phosphatidylinositol and phosphatidylethanolamine (fraction 5), whereas methanol eluted phosphatidylcholine and lysophosphatidylcholine (fraction 6).

The following figures show chromatograms of the same lipid fractions of different wild and cultivated mushrooms. Fig. 7 shows fraction 1, neutral lipids obtained with the Florisil column from Polyporus squamosus, Amanita rubescens, Limacium eburneum, Agaricus silvaticus, A. arvensis and A. bisporus after detection with iodine vapour.

In Fig. 8 the same chromatogram is shown after detection with resorcinol reagent. In Fig. 9 the same chromatogram is detected with the modified Liebermann reagent. Fig. 10 shows the chromatogram of Fig. 9 in UV light.

Fig.7. Isolation of lipid classes on Florisil column with differently polar solvents. I. TLC of fraction 1 .

Indicator: iodine vapour.

Spotted from left to right: Polyporus squamosus, Amanita rubescens, Limacium eburneum, ergosterol, trioleate, Agaricus silvestris, A. arvensis, A. bisporus.

$\begin{array}{lllll}0 & 0 & 0 & 0 \\ 0 & 0 & 0 & 0\end{array}$

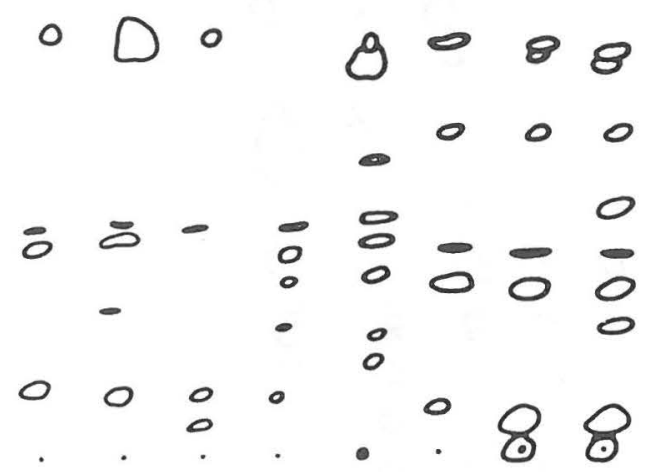

Fig.8. Isolation of lipid classes on Florisil column with differently polar solvents. I. TLC of fraction ?.

Indicator: iodine vapour, resorcinol reagent. Spotted from left to right: Polyporus squamosus, Amanita rubescens, Limacium eburneum, ergosterol, trioleate, Agaricus silvestris, A. arvensis, A. bisporus.

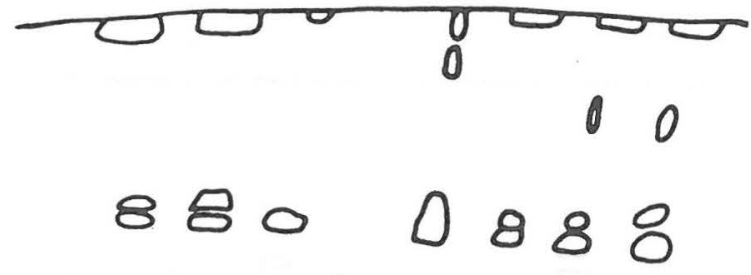

Fig.9.Isolation of lipid classes on Florisil column with differently polar solvents. I. TLC of fraction 1 .

Indicator: Liebermann reagent.

Spotted from left to right: Polyporus squamosus, Amanita rubescens, Limacium eburneum, ergosterol, trioleate, Agaricus silvestris, A. arvensis, A. bisporus.
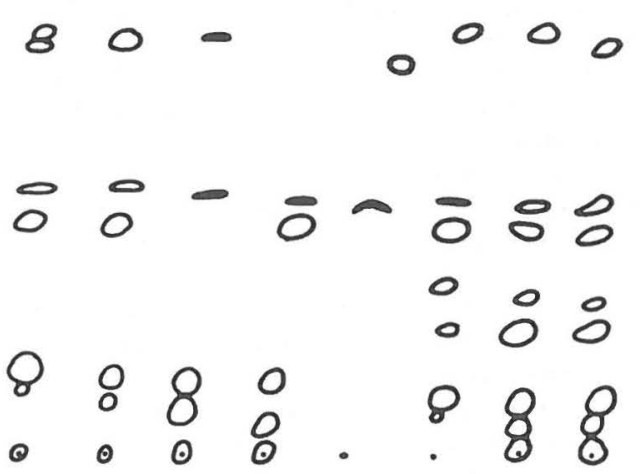
Fig.10.Isolation of lipid classes on Florisil column with differently polar solvents. II. TLC of fraction 2 .

Indicator: Liebermann reagent, illuminated with UV light.

Spotted from left to right: Polyporus squamosus, Amanita rubescens, Limacium eburneum, ergosterol, trioleate, Agaricus silvestris, A. arvensis, A. bisporus.
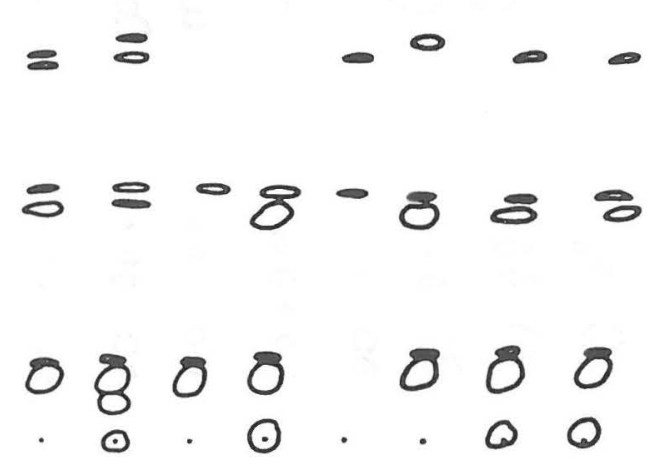

Fig. 11 shows fraction 2 (polar lipids) of the Florisil column detected by the modified Liebermann reagent. The blue spots on the upper part of the chromatogram cannot be detected in the original mushroom extracts; therefore they must be taken for artifacts of the column chromatography.

Fig. 12 shows the carbohydrate-containing components (fraction 3) of C. nebularis, Armillariella mellea (grown on wood), A. mellea (grown on soil), Clitocybe infundibuliformis, Tricholoma nudum and Lepiota procera, detected with resorcinol reagent.

In Fig. 13 is shown the chromatogram of the Florisil column fraction 3. The detection was made first with iodine vapour, and after the sublimation of the iodine with resorcinol reagent spray, heated for some minutes to $100^{\circ} \mathrm{C}$.

Fig. 14 shows the chromatogram of the phosphatidic acid fraction (fraction 4). The detection was made with ammonium molybdate solution (Hanes reagent).

Fig. 15 shows fraction 5 containing phosphatidylserine, phosphatidylinositol and phosphatidylethanolamine, detected with iodine vapour, and Fig. 16 fraction 6 containing phosphatidylcholine and lysophosphatidylcholine, detected with ammonium molybdate. The light blue spots in the lower part of the chromatogram show the lysophosphatidylcholine content of the fungi (Fig. 16).
Fig.11. Isolation of lipid classes on Florisil column with differently polar solvents. II: TLC of fraction 2 .

Indicator: Liebermann reagent.

Spotted from left to right: Polyporus squamosus, Amanita rubescens, Limacium eburneum, ergosterol, trioleate, Agaricus silvestris, A. arvensis, A. bisporus.

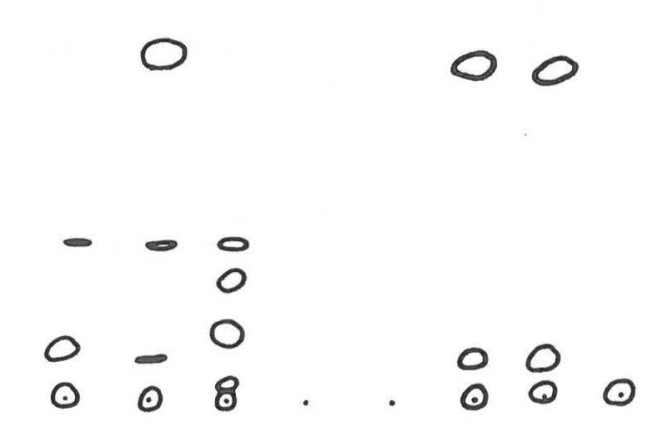

Fig.12. Isolation of lipid classes on Florisil column with differently polar solvents. III. TLC of fraction 3 . Indicator: resorcinol reagent. Spotted from left to right: Clitocybe nebularis, Armizlarielza mellea (grown on wood), A.mellea (grown on soil), ergosterol, tríoleate, clitocybe infundibiliformis, Tricholoma nudum, Lepiota procera.
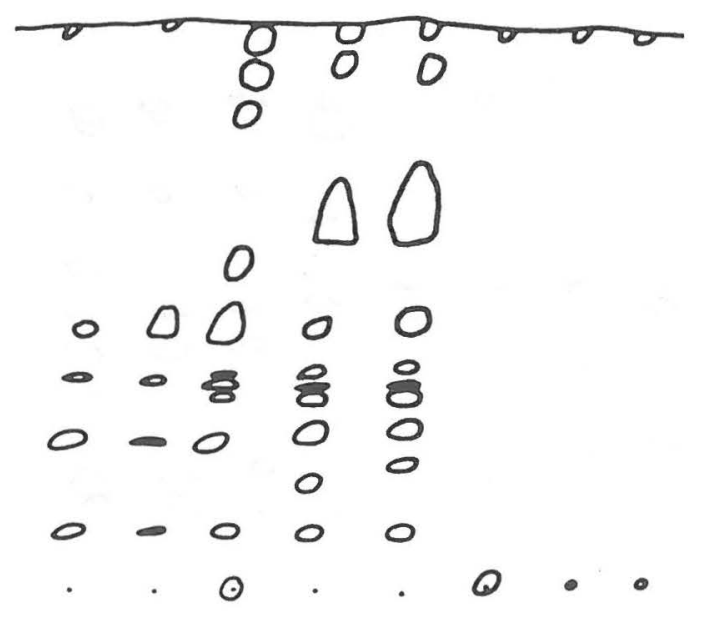
Fig.13.Isolation of lipid classes on Florisil column with differently polar solvents. III. TLC of fraction 3 .

Indicator: iodine vapour, followed by resorcinol reagent.

Spotted from left to right: Polyporus squamosus, Amanita rubescens, Limacium eburneum, ergosterol, trioleate, Agaricus silvestris, A. arvensis, A. bisporus.
Fig.15. Isolation of lipid classes on Florisil column with differently polar solvents. V. TLC of fraction 5 . Indicator: iodine vapour.

Spotted from left to right: Polyporus squamosus, Amanita rubescens, Limacium eburneum, ergosterol, trioleate, Agarious silvestris, A. arvensis,

A. bisporus.

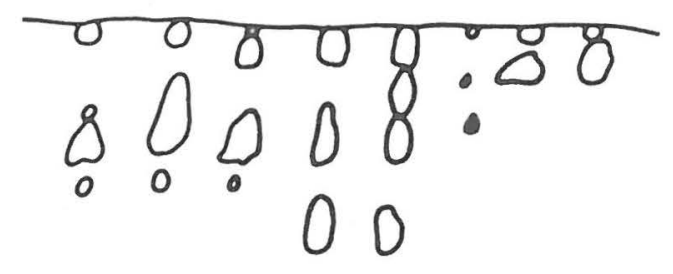

- 0

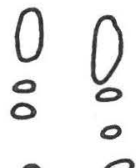

-

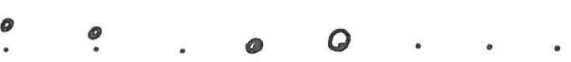

Fig.14.Isolation of lipid classes on Florisil column with differently polar solvents. IV. TLC of fraction 4.

Indicator: Hanes reagent.

Spotted from left to right: Polyporus squamosus Amanita rubescens, Limacium eburneum, ergosterol trioleate, Agaricus silvestris, A. arvensis, A. bisporus.
Fig.16. Isolation of lipid classes on Florisil column with differently polar solvents. VI. TLC of fraction 6 .

Indicator: Hanes reagent.

Spotted from left to right: Clitocybe nebularis, Armillariella mellea (grown on wood), A. mellea (grown on soil), ergosterol, trioleate, Clitocybe infundtbiliformis, Tricholoma nudum, Lepiota procera.

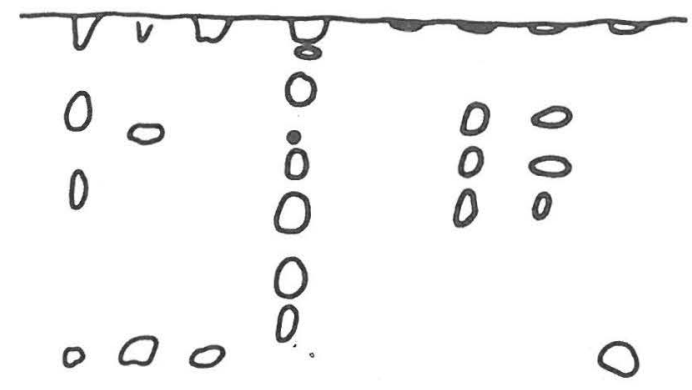<smiles>C1CCCC1</smiles>

$\stackrel{\circ}{\circ} \odot$

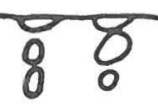

$\begin{array}{ll}0 & 0 \\ 0 & 0 \\ 0 & 0\end{array}$ 


\section{References}

1. Landsiedel, A. \& Bamberger, M. 1905: - Monatsh. Chem. 26: 1109 .

2. Rosenthal, R. 1922: - Monatsh. Chem. 43: 237.

3. Zellner, J. 1911: - Monatsh. Chem. 32: 133, 1057.
4. Hartmann, E. \& Zellner, J. 1928: - Monatsh. Chem. 50: 193 .

5. Froeschl, N. \& Zellner, J. 1928: - Monatsh. Chem. 50: 201 .

6. Brennan, P. J. et al. 1975: - Progress in the Chemistry of Fats and Other Lipids 14: 51.

7. Folch, J., Lees, M. \& Sloane Stanley, G. H. 1957: - J. Biol. Chem. 226: 497. 